\title{
A COMPLETE FROBENIUS TYPE METHOD FOR LINEAR PARTIAL DIFFERENTIAL EQUATIONS OF THIRD ORDER
}

\author{
V. LEÓN* AND B. SCÁRdUA
}

\begin{abstract}
The main subject of this paper is the study of third order linear partial differential equations with analytic coefficients in a two variables domain. We aim the existence of solutions by algorithmic means, in the real or complex analytical case. This is done by introducing methods inspired by the classical method of Frobenius method for analytic second order linear ordinary differential equations. We introduce a notion of Euler type partial differential equation. To such a PDE we associate an indicial cubic, which is an affine plane curve of degree three. Points in this curve are associate to solutions of the Euler PDE. Then comes the concept of regular singularity for the PDE, followed by a notion of resonance and a partial classification of PDEs having such regular singularities. Finally, we obtain convergence theorems, which must necessarily take into account the existence of resonances and the type of PDE (parabolic, elliptical or hyperbolic). We provide some examples of PDEs that may be treated with our methods. This is the first study in this rich subject. Our results are a first step in the reintroduction of techniques from ordinary differential equations in the study of classical problems involving partial differential equations. Our solutions are constructive and computationally viable.
\end{abstract}

Mathematics subject classification (2010): 35A20, 35A24, 35A30, 35C10.

Keywords and phrases: Frobenius method, regular singularity, partial differential equation.

\section{REFERENCES}

[1] A. ARnal And J. Monterde, A third order partial differential equation for isotropic boundary based triangular Bézier surface generation, J. Comput. Appl. Math., 236, 2 (2011), 184-195.

[2] L. Bevilacqua, A. C. N. R. GaleÃo And F. P. Costa, On the significance of higher order differential terms in diffusion processes, J. Braz. Soc. Mech. Sci. \& Eng., 33, 2 (2011), 166-175.

[3] M. I. G. BLOOR AND M. J. Wilson, Generating blend surfaces using partial differential equations, Comput. Aided Des., 21, 3 (1989), 165-171.

[4] D. BorThwick, Introduction to Partial Differential Equations, Universitext, Springer International Publishing, Cham, 2016.

[5] W. E. Boyce And R. C. DiPrima, Elementary Differential Equations and Boundary Value Problems, John Wiley \& Sons, 10th edition, New York, 2012.

[6] E. A. Coddington, An Introduction to Ordinary Differential Equations, Dover Publications, New York, 1989.

[7] M. E. ERdogAn, On unsteady motions of a second-order fluid over a plane wall, Int. J. Non-Linear Mech., 38, (2003), 1045-1051.

[8] G. FrobeniUs, Ueber die Integration der linearen Differentialgleichungen durch Reihen, J. reine angew. Math., 76, (1873), 214-235.

[9] A. HUBER, A novel class of solutions for a non-linear third order wave equation generated by the Weierstrass transformation, Chaos, Solitons \& Fractals, 28, 4 (2006), 972-978.

[10] V. LEÓN AND B. SCÁRDUA, On singular Frobenius for linear differential equations of second and third order, part 1: ordinary differential equations, available at arXiv: 1906.04277v1.

[11] V. LEÓN AND B. SCÁRDUA, On singular Frobenius for second order linear partial differential equations, available at arXiv: 1907.02620v1. 
[12] J. Monterde, The Plateau-Bézier problem, in: Wilson M. J., Martin R. R. (eds), Mathematics of Surfaces, in: Lecture Notes in Comput. Sci., 2768, 262-273, Springer, Heidelberg, 2003.

[13] R. A. VAN GORDER AND K. VAJRAVELU, Third-order partial differential equations arising in the impulsive motion of a flat plate, Commun. Nonlinear Sci. Numer. Simulat., 14, 6 (2009), 2629-2636.

[14] D. A. Weinberg, The affine classification of cubic curves, Rocky Mountain J. Math., 18, 3 (1988), $655-664$. 